\title{
Chronic Myeloid Leukemia: Atypical Presentation and Diagnostic Pitfall in the Workup of Isolated Thrombocytosis
}

Dawood Findakly ${ }^{1}$, Waqas Arslan ${ }^{2,} 3$

1. Internal Medicine, Creighton University Arizona Health Education Alliance/Valleywise Health Medical Center, Phoenix, USA 2. Hematology and Oncology, Creighton University Arizona Health Education Alliance/Valleywise Health, Phoenix, USA 3. Hematology and Oncology, Creighton University Maricopa Medical Center, Phoenix, USA

Corresponding author: Dawood Findakly, dawood_findakly@yahoo.com

\begin{abstract}
Chronic myeloid leukemia (CML) is one of the classic types of myeloproliferative neoplasms. It typically manifests with leukocytosis, but rarely with isolated thrombocytosis. Here we describe a unique case of isolated thrombocytosis as an initial presentation of CML in a 21-year-old woman, where the BCR-ABL1 fusion gene was detected in bone marrow (BM) aspiration and biopsy specimen after a negative peripheral blood (PB) fluorescence in situ hybridization testing. It is crucial to pursue workup for patients with isolated thrombocytosis through testing for the presence of the BCR-ABL fusion gene or the Philadephia chromosome in both $\mathrm{PB}$ and the $\mathrm{BM}$ in order to distinguish CML from essential thrombocythemia.
\end{abstract}

Received 05/24/2020 Review began 05/31/2020 Review ended 05/31/2020 Published 06/07/2020

(c) Copyright 2020 Findakly et al. This is an open access article distributed under the terms of the Creative Commons Attribution License CC-BY 4.0., which permits unrestricted use, distribution, and reproduction in any medium, provided the original author and source are credited.
Categories: Genetics, Internal Medicine, Hematology

Keywords: isolated thrombocytosis, chronic myeloid leukemia, tyrosine kinase inhibitor, bcr-abl positive, philadelphia chromosome

\section{Introduction}

The incidence of chronic myeloid leukemia (CML) in the United States is less than 5,000 cases per year [1]. CML usually presents with marked leukocytosis, but very rarely with an isolated, marked thrombocytosis [2]. Here we present a case of a 21-year-old woman who was discovered to have isolated thrombocytosis incidentally upon evaluation for vasovagal syncope. Her initial peripheral blood (PB) fluorescence in situ hybridization (FISH) testing for the BCR-ABL gene was negative, but subsequent bone marrow (BM) aspiration and biopsy were consistent with CML. This case describes the perplexity in reaching an accurate diagnosis in this subset of patients where diagnosis might be missed and underscores how CML constitutes an essential differential diagnosis in patients presenting with isolated thrombocytosis.

\section{Case Presentation}

A 21-year-old previously healthy woman was referred to the hematology clinic for further workup of thrombocytosis, which was found upon evaluation for vasovagal syncope. Upon her presentation, vital signs were stable and physical examination was unremarkable. Laboratory workup was relevant for a white blood cell (WBC) count of $7.2 \times 10^{\wedge} 3 / \mu \mathrm{L}$, with $56 \%$ neutrophils, $33 \%$ lymphocytes, $7 \%$ monocytes, $1 \%$ basophils, and $0.2 \%$ eosinophils, hemoglobin of $13.3 \times 10^{\wedge} 3 / \mu \mathrm{L}$, platelets (PLT) of $764 \mathrm{~K} / \mu \mathrm{L}$, and minimally elevated erythrocyte sedimentation rate at $39 \mathrm{~mm} / \mathrm{hr}$. Peripheral smear confirmed thrombocytosis, and the patient was started on daily low-dose aspirin (Figure 1). 


\section{Cureus}

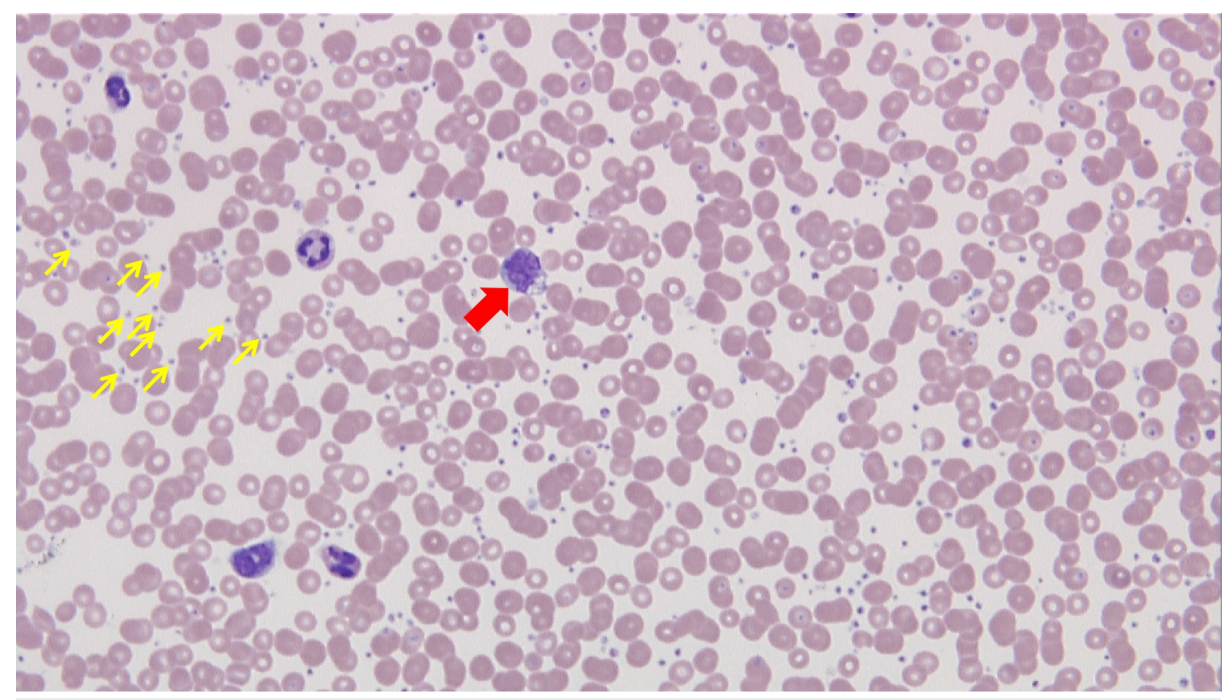

FIGURE 1: The PB smear exhibits markedly increased PLT (yellow arrows), anisopoikilocytosis without evidence of eosinophilia or basophilia. A rare immature monocyte displayed (red arrow) could rarely be seen.

PB: peripheral blood; PLT: platelet

Meanwhile, the thrombocytosis workup for myeloproliferative disorders (MPDs) with PB JAK2 on exon 12 and 14, calreticulin (CALR), and thrombopoietin receptor (MPL) genes were not mutated. Moreover, PB FISH for BCR-ABL was negative, and a CT scan of the abdomen and pelvis was performed and was unremarkable. As PLT count continued to rise, reaching $1,096 \mathrm{~K} / \mu \mathrm{L}$, the hematology consultant recommended bone marrow aspirate and biopsy; which revealed overall cellularity of approximately $65 \%$ $70 \%$ with $14 \%$ segmented neutrophils, $10 \%$ bands, $7 \%$ metamyelocytes, $10 \%$ myelocytes, $0 \%$ progranulocytes, $3 \%$ blasts, $3 \%$ monocytes, $4 \%$ eosinophils, $0 \%$ basophils, $16 \%$ lymphocytes, $2 \%$ plasma cells, and 28\% nucleated red blood cells (Figure 2). Moreover, bone marrow molecular biology analysis of the $\mathrm{BCR} / \mathrm{ABL}$ fusion gene revealed a translocation $\mathrm{t}(9 ; 22) \mathrm{BCR}-\mathrm{ABL} 1$ consistent with CML.
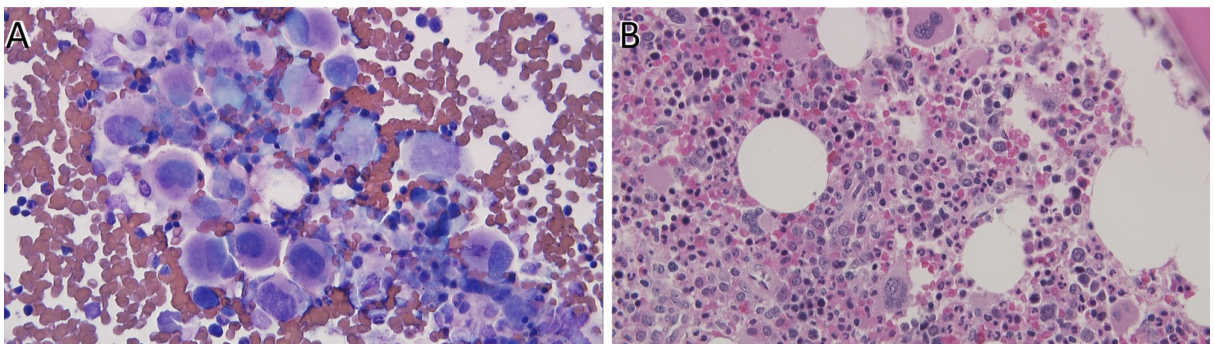

FIGURE 2: (A) BM aspirate smear; (B) BM core biopsy demonstrating an overall cellularity of approximately $65 \%-70 \%$. The megakaryocytes are markedly increased with clustered numerous small and unilobed megakaryocytes appreciated. Moreover, the myeloid to erythroid ratio is at approximately $3: 1$ with a relative decrease in erythroid precursors.

BM: bone marrow

Subsequently, the patient was initiated on a second-generation tyrosine kinase inhibitor (TKI), dasatinib, achieving a complete cytogenetic and molecular remission (Figure 3). The patient continues in remission at 32 months follow-up and is being evaluated for stem cell transplant. 


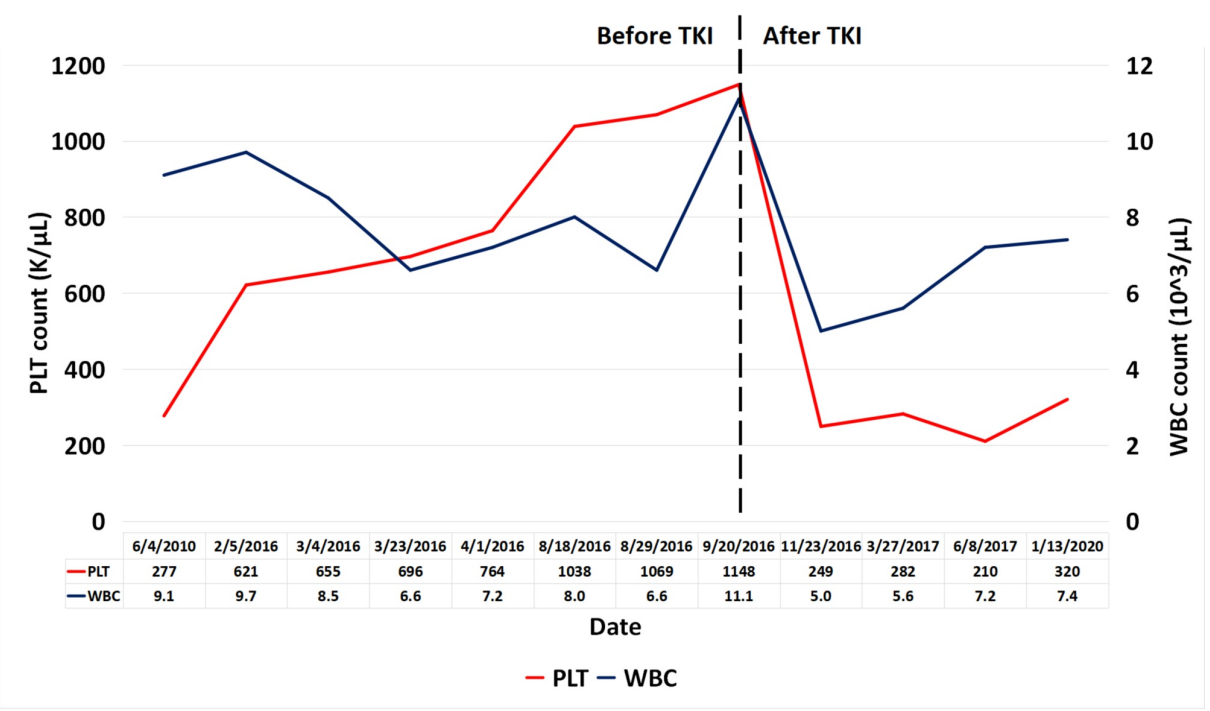

FIGURE 3: Timeline for PLT and WBC trend during follow-up, before and after TKI (dasatinib) therapy. The $\mathrm{x}$-axis constitutes the date when testing was performed, the left $y$-axis constitutes PLT count, and the right $y$-axis constitutes WBC count. The vertical black hatched line indicates the starting point of TKI (dasatinib) treatment.

PLT: platelet; WBC: white blood cell; TKI: tyrosine kinase inhibitor

\section{Discussion}

CML incidence in the United States is approximately 1.3 cases per 100,000 per year [1]. Patients with CML are, in many cases, asymptomatic at diagnosis and only incidentally detected upon abnormalities found on routine blood testing. CML is the most common condition in the spectrum of MPDs [3]. It typically presents with leukocytosis, with elevated immature granulocytes, basophilia, and eosinophilia in the PB [3,4].

This atypical CML presentation with an isolated thrombocytosis is a rare entity [5]. Isolated thrombocytosis could lead to a misdiagnosis of essential thrombocythemia (ET) [4]. Thrombocytosis, described as a platelet count of more than $450 \times 10^{9} / \mathrm{L}$, is common in chronic MPDs [3]. Thrombocytosis could be reactive or it may be paraneoplastic where it could be triggered by altered immune response to MPDs, including polycythemia vera, CML, or ET $[4,6]$. Severe thrombocytosis, with a platelet count of more than $1,000 \times 10^{9} / \mathrm{L}$, is exceptionally rare to be found upon initial laboratory workup in newly diagnosed patients with CML [3]. Every patient presenting with isolated thrombocytosis should be tested to rule out CML [7]. CML is characterized by persistently enhanced Philadelphia positive $(\mathrm{Ph}+)$ chromosome, which is an active tyrosine kinase [8,9]. BCR-ABL has tyrosine kinase activity, which promotes cell proliferation and enhances resistance to apoptosis [9].

Despite the increasing prevalence of CML cases, TKI chemotherapy remains successful in improving survival outcomes [10]. BM specimens are superior to PB specimens in detecting BCR-ABL fusions [11]. Atypical CML presentation, with isolated thrombocytosis, should be considered in patients presenting with isolated marked thrombocytosis, as it signifies a very poor prognosis with a median overall survival of 24 months [12].

\section{Conclusions}

Given its reduced survival outcomes, atypical presentation of CML should remain high in the differential diagnosis when evaluating patients with significant thrombocytosis, even when WBC count is normal. These patients should not be misdiagnosed as ET, and providers should pursue genetic testing for the BCR/ABL gene in both PB and BM in order to circumvent delayed or missed diagnosis, which could ultimately interfere with optimal medical treatment and prognosis. Further research is necessary to evaluate the clinicopathologic characteristics, treatments, and outcomes of CML patients presenting with isolated marked thrombocytosis.

\section{Additional Information}




\section{Disclosures}

Human subjects: Consent was obtained by all participants in this study. Conflicts of interest: In compliance with the ICMJE uniform disclosure form, all authors declare the following: Payment/services info: All authors have declared that no financial support was received from any organization for the submitted work. Financial relationships: All authors have declared that they have no financial relationships at present or within the previous three years with any organizations that might have an interest in the submitted work. Other relationships: All authors have declared that there are no other relationships or activities that could appear to have influenced the submitted work.

\section{References}

1. Huang X, Cortes J, Kantarjian H: Estimations of the increasing prevalence and plateau prevalence of chronic myeloid leukemia in the era of tyrosine kinase inhibitor therapy. Cancer. 2012, 118:3123-3127. 10.1002/cncr.26679

2. Thompson PA, Kantarjian HM, Cortes JE: Diagnosis and treatment of chronic myeloid leukemia in 2015 . Mayo Clin Proc. 2015, 90:1440-1454. 10.1016/j.mayocp.2015.08.010

3. Liu Z, Fan H, Li Y, Liu C: Analysis of clinical characteristics and efficacy of chronic myeloid leukemia onset with extreme thrombocytosis in the era of tyrosine kinase inhibitors. Onco Targets Ther. 2017, 10:35153520. 10.2147/OTT.S142587

4. Okano M, Sugimoto Y, Ohishi K, Miyazaki K, Monma F, Katayama N: Isolated thrombocytosis in chronic myeloid leukemia without significant leukocytosis [Article in Japanese]. Rinsho Ketsueki. 2017, 58:766-771. 10.11406/rinketsu.58.766

5. Kodali D, Mesa H, Rawal A, Cao Q, Gupta P: Thrombocytosis in myelodysplastic and myelodysplastic/myeloproliferative syndromes. Leuk Lymphoma. 2007, 48:2375-2380. 10.1080/10428190701724827

6. Bleeker JS, Hogan WJ: Thrombocytosis: diagnostic evaluation, thrombotic risk stratification, and risk-based management strategies. Thrombosis. 2011, 2011:536062. 10.1155/2011/536062

7. Rice L, Popat U: Every case of essential thrombocythemia should be tested for the Philadelphia chromosome. Am J Hematol. 2005, 78:71-73. 10.1002/ajh.20257

8. An X, Tiwari AK, Sun Y, Ding PR, Ashby CR Jr, Chen ZS: BCR-ABL tyrosine kinase inhibitors in the treatment of Philadelphia chromosome positive chronic myeloid leukemia: a review. Leuk Res. 2010, 34:1255-1268. 10.1016/j.leukres.2010.04.016

9. Kang ZJ, Liu YF, Xu LZ, et al.: The Philadelphia chromosome in leukemogenesis . Chin J Cancer. 2016, 35:48. 10.1186/s40880-016-0108-0

10. Pophali PA, Patnaik MM: The role of new tyrosine kinase inhibitors in chronic myeloid leukemia . Cancer J. 2016, 22:40-50. 10.1097/PPO.0000000000000165

11. Manaflouyan Khajehmarjany S, Rahmani SA, Chavoshi SH, Esfahani A, Movassaghpour Akbari AA: Reliability evaluation of fluorescence in situ hybridization (FISH) and G-banding on bone marrow and peripheral blood cells in chronic myelogenous leukemia patients. Cell J. 2015, 17:171-180. 10.22074/cellj.2015.525

12. Drozd-Sokołowska JE, Waszczuk-Gajda A, Mądry K, Dwilewicz-Trojaczek J: Atypical chronic myeloid leukaemia: a rare subtype of myelodysplastic/myeloproliferative neoplasm. Contemp Oncol. 2018, 22:14-19. 10.5114/wo.2018.74388 